\title{
CRITERIAADOPTED BY DENTISTS TO INDICATE THE EXTRACTION OF PERIODONTALLY INVOLVED TEETH
}

\author{
Carlos Heitor Cunha MOREIRA ${ }^{1}$, Fabricio Batistin ZANATTA ${ }^{2}$, Raquel ANTONIAZZI ${ }^{3}$, \\ Priscila Ceolin MENEGUETTI ${ }^{4}$, Cassiano Kuchenbecker RÖSING ${ }^{5}$
}

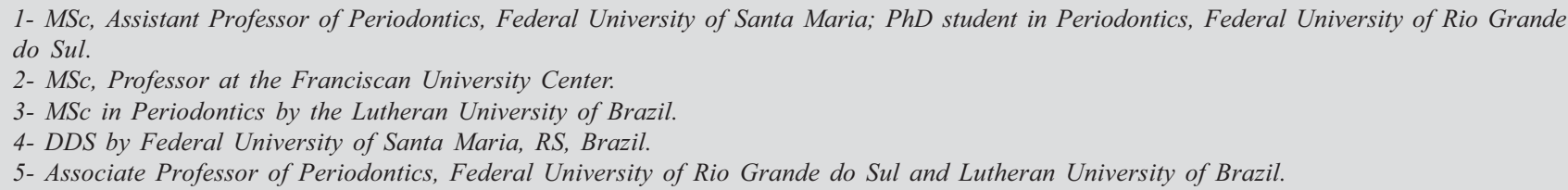

\begin{abstract}
$W$ hen dealing with patients with periodontal disease of variable severities, dentists must often choose between treating and restoring the involved tooth or indicating its extraction. Different criteria have been adopted in this decision-making process. The purpose of this study was to evaluate the criteria adopted by dentists to indicate the extraction of teeth with periodontitis. Dentists were interviewed at their private practices in three cities of the state of Rio Grande do Sul, Brazil. The evaluated criteria included severity of attachment loss, tooth mobility, furcation involvement, prosthetic planning, periodontal-endodontic lesion, possible systemic involvement due to the presence of periodontitis, referral to a periodontist for evaluation, radiographic bone loss greater than $50 \%$, presence of extensive caries, socio-economic and cultural status of the patient, among others. The most often adopted criteria to indicate the extraction of periodontally affected teeth were the presence of mobility (37.5\%), severity of attachment loss $(24.3 \%)$ and radiographic bone loss greater than $50 \%(21.2 \%)$. The results of the present study demonstrated the difficulties faced by dentists to indicate the extraction of teeth with severe attachment loss, in addition to the establishment of an adequate prognosis. Aspects associated with the past disease were still the most often reported to indicate the extraction of teeth for periodontal reasons.
\end{abstract}

Uniterms: Prognosis; Periodontal disease; Tooth extraction.

\section{INTRODUCTION}

Periodontal diseases are highly prevalent in populations ${ }^{27}$. Epidemiological studies have demonstrate variable distributions in different populations and reveal that this is the second greatest cause of tooth loss ${ }^{1,28}$.

The main goal of periodontal treatment is to reestablish the balance of periodontal health, restoring the health and function of teeth ${ }^{23}$.

Treatment of advanced periodontal disease aiming to control the supra and subgingival biofilm has a favorable prognosis. It is suggested that, if the supragingival biofilm is well controlled subsequently to treatment, the outcomes are adequately maintained and future attachment loss is prevented ${ }^{10,11,14,24}$. Moreover, patients attending a maintenance

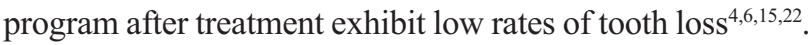
However, in some situations, the previous attachment loss may impair the achievement of such therapeutic goals, and health and function of teeth may not be reestablished by periodontal treatment. In these situations, extraction of teeth with periodontal disease is recommended.

Prognosis is based on the diagnosis and therapeutic possibilities according to the duration, evolution and resolution of the disease ${ }^{7}$. The prognosis of periodontally compromised teeth depends on the analysis of specific data referring to the disease and the individual teeth, such as distribution, type and degree of bone loss, probing depth, attachment loss, presence and severity of furcation lesions, mobility, crown-root relationship, root anatomy, pulp involvement, position and occlusal relationship of the tooth, type of rehabilitation to be adopted, strategic value of the tooth, in addition to factors related to the patient, such as age, systemic status, oral hygiene, other risk factors and the possibility to change them, financial aspects, parafunctional habits and follow-up intervals s, $^{3,20,23}$.

It is difficult to establish the prognosis of periodontally 
treated teeth without good conditions. These difficulties seem to be greater for teeth initially classified as questionable, especially molars ${ }^{16}$. The inclusion of purely anatomical aspects in most reported categories of prognosis is one of the factors worsening the difficulties of proper prediction of periodontal therapy. Analysis of patient's expectations, motivation and change in habits as to the therapy performed, presence and possibility of risk factors and occasional rehabilitation treatments are important in periodontal planning and prognosis yet are often disconsidered ${ }^{12,26}$.

The preference for a certain procedure depends on several factors, including technical-scientific knowledge, experience, tradition, beliefs and habits. Considering the fragility of such aspects, the clinical proposals should be based on wellestablished aspects, encouraging the modern dental professionals to permanently reconsider and update their knowledge.

When caring for patients with periodontal disease of variable severity, dental professionals must often choose between treating and restoring the involved tooth or indicating its extraction; different criteria have been adopted in this decision-making process. Establishing an adequate prognosis is a difficult task. Knowing the advantages and limitations of the criteria is of great importance for clinicians and college teachers, especially because ethical question arises for the decision of maintaining teeth. It is of utmost interest to evaluate which are the currently adopted criteria, in order to subsidize continuing education.

The aim of the present study was to evaluate, by means of interviews, the criteria adopted by dentists to indicate the extraction of periodontally involved teeth.

\section{MATERIALAND METHODS}

Data were collected by means of interviews with dentists working at the cities of Santa Maria, Cruz Alta and Rosário do Sul, in the state of Rio Grande do Sul, Brazil. Professionals were identified with aid of records supplied by the Regional Dental Council of the state of Rio Grande do Sul. The interviews with dentists from the city of Santa Maria were classified into two strata, according to the year of graduation and location of the private practice, and were randomly drafted among the dental professionals registered in the Regional Dental Council.
Contact was attempted with all professionals at the other two cities; if the interview was not possible after 3 attempts, the next consecutive dental professional registered in the Regional Dental Council was interviewed. In Santa Maria, 27 dentists had moved from the registered address, and 22 were not found after 3 attempts. In Cruz Alta, 14 had moved and 25 others were not found after 3 attempts. In the city of Rosário do Sul, 1 dentist had retired, 1 had moved away and 5 were not found after 3 attempts. None of the contacted dentists denied participating. All interviews were performed at their private practices.

Before the interview, the study subjects were informed on the objectives of the study and the privacy of data. Then, the dentists willing to participate in the study signed an informed consent form. The study design was reviewed and approved by the Institutional Review Board of the Federal University of Santa Maria.

Table 1 presents the sample of dentists interviewed, according to the city and year of graduation.

A pilot study was conducted on 10 dentists to evaluate the questions employed in the interviews and the time required for their application. Information obtained from these interviews was used to adjust the questions.

A total of 152 dental professionals were interviewed, being 108 at Santa Maria, 29 at Cruz Alta and 15 at Rosário do Sul. The year of graduation ranged from 1960 to 2005, with median at the year 1982. Most dentists were general practitioners: $52.8 \%$ at Santa Maria, $75.9 \%$ at Cruz Alta and $93.3 \%$ at Rosário do Sul.

Open and closed questions were employed in the interview. More than one answer applied to each question. The alternatives related to the criteria adopted to indicate the extraction of teeth with periodontitis included severity of attachment loss, tooth mobility, furcation involvement, prosthetic planning, periodontal-endodontic lesion, possible systemic involvement due to periodontitis, referral to a periodontist for evaluation, radiographic bone loss greater than $50 \%$, presence of extensive caries, socio-economic and cultural status of the patient, among others.

The results were analyzed by frequency distribution of responses to the interviews. These distributions were stratified according to the year of graduation. The dentists were stratified by year of graduation in three strata (1960$1975,1976-1990,1991-2005)$ The use of the most related

TABLE 1- Number and percentage $(n(\%))$ of dentists interviewed in the study

\begin{tabular}{llll}
\hline & Santa Maria & Cruz Alta & Rosário do Sul \\
\hline Total number of dentists & $592(100)$ & $68(100)$ & $22(100)$ \\
Dentists interviewed & $108(18.24)$ & $29(42.64)$ & $15(68.18)$ \\
$1960-1970$ & $11(10.18)$ & $6(20.68)$ & $4(26.66)$ \\
$1971-1980$ & $34(31.48)$ & $7(24.13)$ & $4(26.66)$ \\
$1981-1990$ & $23(21.29)$ & $7(24.13)$ & $2(13.33)$ \\
$1991-2000$ & $34(31.48)$ & $9(31.03)$ & $4(26.66)$ \\
$2001-2005$ & $6(5.55)$ & $0(0)$ & $1(6.66)$ \\
\hline
\end{tabular}


parameters was tested among these strata by chi-square test. The level of significance was set at $5 \%$.

\section{RESULTS}

Fifty-five percent of the dentists reported that they refer their patients to a periodontist to determine whether periodontally affected teeth should be extracted.

The most frequently reported reasons to indicate the extraction of teeth with periodontitis were the presence of mobility (37.5\%), severity of attachment loss (24.3\%) and radiographic bone loss greater than $50 \%(21.2 \%)$, followed by socioeconomic and cultural aspects $(16.4 \%)$, prosthetic planning (12.5\%) and furcation involvement (5.3\%). Reasons such as presence of extensive caries (3.3\%), possibility of systemic involvement due to periodontitis $(3.3 \%)$ and periodontal-endodontic lesions $(2.0 \%)$ were the least mentioned among the reasons for tooth extraction (Table 2). Figure 1 presents the reasons mentioned for tooth extraction, with stratification according to the year of graduation.

When the subgroups relating the time of graduation were analyzed, it was observed that the earlier graduates referred to periodontists more frequently to establish prognosis $(\mathrm{p}=0.008)$. Regarding mobility and severity of loss of attachment as reasons to tooth extraction, no differences were observed regarding time after graduation $(p>0.05)$

\section{DISCUSSION}

The present study interviewed dental professionals on the decision-making process to indicate the extraction of periodontally compromised teeth. So far, it is not possible to adequately predict the risk of future attachment loss, or if a patient without attachment loss may or may not be a patient at risk in the future, due to several variables that might be present later in life ${ }^{8,18}$. However, patients without history of periodontitis seem to be at lower risk to become susceptible compared to patients previously affected by periodontitis ${ }^{24}$. The presence of risk factors, evaluation of susceptibility, as well as factors that affect the prognosis, should be considered in clinical decision-making involving the indication for extraction $^{17}$. The possibility of healing in periodontics and maintenance of treated teeth for long time periods is currently well established ${ }^{4,9}$. However, in some cases, the severity of destruction of periodontal tissues does not provide conditions for healing; thus, tooth extraction should be indicated.

In the present study, a high percentage of interviewees reported that they refer their patients to a periodontist to evaluate the possibility of treatment or indicate the extraction of teeth with periodontitis. More experienced dentists were the ones referring the most. One could infer that experience in clinical practice is associated with narrowing the clinical activity, referring more frequently to specialists. On the other hand, this aspect seems to demonstrate the daily clinical difficulties involved in treatment planning for patients with advanced periodontitis. Establishment of proper and predictable prognosis in Periodontics is limited ${ }^{16}$. However, even teeth with doubtful prognosis at treatment onset may have a better prognosis after adequate treatment ${ }^{16}$.

Tooth mobility, severity of attachment loss and radiographic bone loss greater than $50 \%$, in this order, were the most frequently adopted criteria to indicate the extraction of periodontally affected teeth. These criteria indicate the severity of disease, as well as its sequelae after treatment. The severity of attachment loss was the most reported by recently graduated dental professionals, whereas evaluation of radiographic bone loss was the most reported by dental professionals graduated between 1960-1970. With regard to the presence of mobility, there was no difference among dentists graduated at different times. The presence of attachment loss is also a predictor of future risk of attachment loss, and thus it is adopted to estimate the patient's susceptibility. The presence of mobility, though disturbing for both dental professionals and patients, should not be the determining factor to indicate tooth extraction because it may

TABLE 2- Criteria adopted by the dentists to indicate the extraction of teeth with periodontitis $(n=152)$

\section{Criteria}

Severity of attachment loss

Tooth mobility

Furcation involvement

Prosthetic planning

Periodontal-endodontic lesion

Possible systemic involvement due to the presence of periodontitis

Referral to a periodontist for evaluation

Radiographic bone loss greater than $50 \%$

Presence of extensive caries

Socio-economic and cultural status of the patient

Other criteria

Yes n Percentage (\%)

37

57

8

19

3

5

85

32

5

25

26
24.3

37.5

5.3

12.5

2.0

3.3

55.9

21.2

3.3

16.4

17.1 
be reduced by a decrease in the inflammatory infiltrate after treatment, leading to stability in the long term ${ }^{2}$. Radiographs are helpful to determine the diagnosis and prognosis in Periodontics and should be examined as to the presence of other pathologies, bone crest level, inter-radicular bone resorption, and crown-root ratio. The limitations of radiographic examination for analysis of the topography of bone defects and establishment of the periodontal health status should be mentioned ${ }^{5}$. These three criteria (severity of attachment loss, tooth mobility and radiographic bone loss) are consequences of the past periodontal disease and reflect the severity of present tissue destruction upon examination ${ }^{13}$.

The severity of attachment loss does not indicate the activity of disease upon examination. This may only be determined by the presence of clinical attachment loss or radiographic bone loss evaluated in 2 examinations at different moments. The presence of clinical inflammatory signs such as edema, erythema, bleeding and suppuration indicate the inflammatory status and, if considered separately, are weak predictors of future attachment loss ${ }^{3}$.

Prosthetic planning was also indicated as a criterion adopted to indicate the extraction of teeth with periodontitis. Dental professionals who graduated earlier reported this criterion more frequently for evaluation of prognosis. Even teeth with reduced periodontal attachment may be used as prosthetic abutments ${ }^{21}$.
The systematic evaluation of furcation involvement was not frequently mentioned in the interviews. More recently, the prognosis of teeth with furcation involvement has been re-considered, and furcation involvement per se is not currently considered a determining factor in the establishment of prognosis ${ }^{29}$. The present study did not aim to evaluate if dental professionals analyze the presence or not of furcation involvement. The low frequency of this criterion may indicate the lack of diagnosis of furcation lesions. Also, the presence of periodontal-endodontic lesions was not frequently considered for the establishment of prognosis. Determination of the primary origin of the lesion, as well as the possibility of repair associated with endodontic and periodontal treatment, are associated with the possibility of healing ${ }^{25}$.

Socioeconomic and cultural aspects may be related with the wish and possibility of patients to undergo certain types of dental treatment ${ }^{26}$. The dental professional should consider different possible treatment options for the effective treatment of patients. However, the patients always have the right to receive all information on the therapeutic options available to solve their pathologies.

The presence of carious lesions and the possibility of systemic involvement due to the presence of periodontitis had a low frequency of positive responses. The possibility of resolution of extensive carious lesions by restorative procedures, as well as the possibility of resolution of

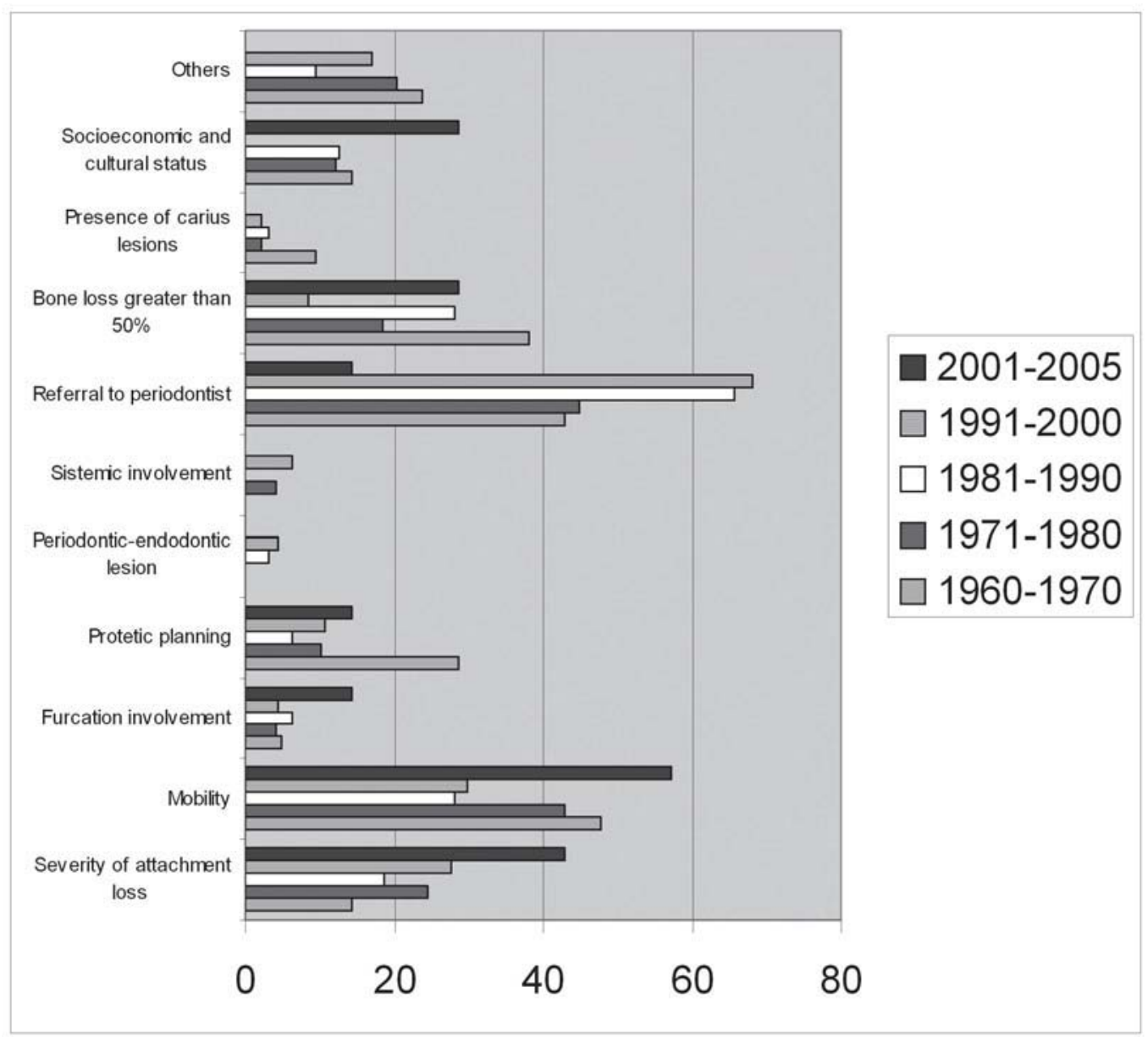

FIGURE 1- Criteria adopted by the dentists to indicate the extraction of teeth with periodontitis (\%), stratified according to year of graduation 
inflammation associated with periodontal diseases by proper treatment, may reflect these results ${ }^{4,19}$. The concept of "Periodontal Medicine" was introduced in the early 1990s, and not all interviewees may be aware of the important association between periodontal disease and systemic conditions $^{30}$.

Predictable criteria should be developed to allow the establishment of correct prognosis for patients with periodontitis. Use of anatomical criteria exclusively, without no reference to the biological status and presence of risk factors, may jeopardize the accuracy of the established prognosis.

\section{CONCLUSION}

It may be concluded that establishing prognosis is a difficulty faced by the dentists, which was reflected by the high percentage of referral to specialists to establish prognosis. Past disease experience accounted for the highest percentage of indications for extraction. Dentists still need more reliable methods to establish prognosis and preserve the teeth.

\section{REFERENCES}

1- Albandar JM, Rams TE. Global epidemiology of periodontal diseases: an overview. Periodontol 2000. 2002;29(1):7-10

2- Anderegg C, Metzler DG. Tooth mobility revisited. J Periodontol. 2001;72(7):963-7.

3- Armitage GC. Periodontal diseases: diagnosis. Ann Periodontol. 1996;1(1):37-215.

4- Axelsson P, Nystrom B, Lindhe J. The long-term effect of a plaque control program on tooth mortality, caries and periodontal disease in adults. Results after 30 years of maintenance. J Clin Periodontol. 2004;31(9):749-57.

5- Brägger U. Radiographic parameters: biological significance and clinical use. Periodontol 2000. 2005;39(1):73-81

6- Checchi L, Montevecchi M, Gatto MR, Trombelli L. Retrospective study of tooth loss in 92 treated periodontal patients. J Clin Periodontol. 2002;29(7):651-6

7- Cortelli JR, Lotufo RMF, Oppermann RV, Sallum AW, Costa FO. Glossário da Sociedade Brasileira de Periodontologia. Periodontia. 2005;15(4):3-61.

8- Greenstein G, Hart TC. Clinical utility of a genetic susceptibility test for severe chronic periodontitis: a critical evaluation. J Am Dent Assoc. 2002;133(4):452-9.

9- Greenstein G. Periodontal diseases are curable. J Periodontol. $2002 ; 73(8): 950-3$

10 - Haffajee AD, Cugini MA, Dibart S, Smith C, Kent RL JR, Socransky SS. The effect of SRP on the clinical and microbiological parameters of periodontal diseases. J Clin Periodontol. 1997;24(5):324-34.

11 - Kaldahl WB, Kalkwarf KL, Patil KD, Molvar MP, Dyer JK. Longterm evaluation of periodontal therapy: II. Incidence of sites breaking down. J Periodontol. 1996;67(2):103-8.
12- Kinane DF, Peterson M, Stathopoulou PG. Environmental and other modifying factors of the periodontal diseases. Periodontol 2000. 2006;40(1):107-19.

13 - Lang NP, Tonetti MS. Periodontal diagnosis in treated periodontitis: why, when and how to use clinical parameters. J Clin Periodontol. 1996,23(3 Pt 2):240-50.

14- Lindhe J, Westfelt E, Nyman S, Socransky SS, Haffajee AD. Longterm effect of surgical/non-surgical treatment of periodontal disease. J Clin Periodontol. 1984;11(7):448-58

15- McFall WT Jr. Tooth loss in 100 treated patients with periodontal disease. A long-term study. J Periodontol. 1982;53(9):539-49.

16- McGuire MK. Prognosis versus actual outcome: a long-term survey of 100 treated periodontal patients under maintenance care. J Periodontol. 1991;62(1):51-8.

17- McGuire MK. Prognosis vs outcome: predicting tooth survival. Compend Contin Educ Dent. 2000;21(3):217-20, 222, 224.

18 - Mombelli A. Critical issues in periodontal diagnosis. Periodontol 2000. 2005;39(1):9-21.

19- Morgano SM, Brackett SF. Foundation restorations in fixed prosthodontics: current knowledge and future needs. J Prosthet Dent. 1999;82(6):643-57.

20-Needleman I, Moles DR, Worthington H. Evidence-based periodontology, systematic reviews and research quality. Periodontol 2000. 2005;37(1):12-20.

21 - Nyman S, Lindhe J. A longitudinal study of combined periodontal and prosthetic treatment of patients with advanced periodontal disease. J Periodontol. 1979;50(4):163-9.

22- Papapanou PN, Wennstrom JL. A 10-year retrospective study of periodontal disease progression. Clinical characteristics of subjects with pronounced and minimal disease development. J Clin Periodontol. 1990;17(2):78-84

23 - Ramfjord SP. Maintenance care and supportive periodontal therapy. Quintessence Int. 1993;24(7):465-71.

24- Rosling B, Serino G, Hellstrom MK, Socransky SS, Lindhe J. Longitudinal periodontal tissue alterations during supportive therapy. Findings from subjects with normal and high susceptibility to periodontal disease. J Clin Periodontol. 2001;28(3):241-9.

25 - Rotestein I, Simon JH. Diagnosis, prognosis and decision-making in the treatment of combined periodontal-endodontic lesions. Periodontol 2000. 2004;34:165-203.

26- Sheiham A, Nicolau B. Evaluation of social and psychological factors in periodontal disease. Periodontol 2000. 2005;39(1):118-27.

27- Susin C, Dalla Vecchia CF, Oppermann RV, Haugejorden O, Albandar JM. Periodontal attachment loss in an urban population of Brazilian adults: the effect of demographic, behavioral an environmental risk indicators. J Periodontol. 2004;75(7):1033-41.

28- Susin C, Oppermann RV, Haugejorden O, Albandar JM. Tooth loss and associated risk indicators in an adult urban population from south Brazil. Acta Odontol Scand. 2005;63(2):85-93.

29- Svardstrom G, Weenstrom JL. Periodontal treatment decisions for molars: an analysis of influencing factors and long-term outcome. J Periodontol. 2000;71(4):579-85.

30 - Williams RC, Offenbacher S. Periodontal medicine: the emergence of a new branch of periodontology. Periodontol 2000. 2000;23:9-12. 